\title{
The Hardy-Rellich Inequality for Polyharmonic Operators
}

\author{
M. P. OWEN \\ Department of Mathematics, King's College London, Strand, London, WC2R 2LS
}

\begin{abstract}
The Hardy-Rellich inequality given here generalizes a Hardy inequality of Davies [2], from the case of the Dirichlet Laplacian of a region $\Omega \subseteq \mathbb{R}^{N}$ to that of the higher order polyharmonic operators with Dirichlet boundary conditions. The inequality yields some immediate spectral information for the polyharmonic operators and also bounds on the trace of the associated semigroups and resolvents.
\end{abstract}

\section{INTRODUCTION}

The Hardy inequality originated in 1920 in [7] as an integral inequality for functions defined on the real half-line. Its original representation can be easily reformulated, for $1<p<\infty$, as

$$
\int_{0}^{\infty} \frac{|f(x)|^{p}}{x^{p}} \mathrm{~d} x \leq\left(\frac{p}{p-1}\right)^{p} \int_{0}^{\infty}\left|f^{\prime}(x)\right|^{p} \mathrm{~d} x
$$

for all $f \in C_{c}^{\infty}((0, \infty))$. Since its appearance, various generalizations of particular aspects of the inequality have been made. In [10], for example, there is a detailed treatment of weighted Hardy-type inequalities in an $L^{p}$ setting.

The Rellich inequality appeared first in [13 as a generalization of inequality (11) to two derivatives. The simplest form of such an inequality is

$$
\int_{0}^{\infty} \frac{|f(x)|^{p}}{x^{2 p}} \mathrm{~d} x \leq\left(\frac{p^{2}}{(p-1)(2 p-1)}\right)^{p} \int_{0}^{\infty}\left|f^{\prime \prime}(x)\right|^{p} \mathrm{~d} x
$$

for all $f \in C_{c}^{\infty}((0, \infty))$.

In this paper we study a generalization for all derivatives, within the $L^{2}$ setting. The variable $x$ in the denominator of inequalities (11) and (2) is replaced by a pseudodistance $a_{m}(x)$, where $m$ is the number of derivatives in 
the dominating integrand. We formulate our result as a Hardy-Rellich operator inequality, and use it as a tool in the spectral analysis of polyharmonic operators. The Rellich inequalities found in [6] concern a distinct but related class of operator inequalities.

In order to state our result properly, we need the following definitions, in which $\Omega$ denotes an open subset of $\mathbb{R}^{N}$ :

Definition 1: Let $Q_{m}$ be the closure of the quadratic form defined on $C_{c}^{\infty}(\Omega) \subseteq$ $L^{2}(\Omega)$ by

$$
Q_{m}(f)=\left\langle(-\Delta)^{m} f, f\right\rangle .
$$

The domain of the closure is the Sobolev space $W_{0}^{m, 2}(\Omega)$. The polyharmonic operator $\left.(-\Delta)^{m}\right|_{\mathrm{DIR}}$ is defined as the non-negative self-adjoint operator associated with $Q_{m}$. See [5] for details. Where the implied region is not contextually evident, the operator is denoted by $H_{\Omega, m}$.

The boundary conditions classically associated with the operators $\left.(-\Delta)^{m}\right|_{\text {DIR }}$ and $\left(-\left.\Delta\right|_{\text {DIR }}\right)^{m}$ are different. The inequality

$$
H_{\Omega, m} \geq H_{\Omega, 1}^{m}
$$

may be verified by considering quadratic form domains.

Definition 2: Let $\omega \in S^{N-1}$ and define $d_{\omega}: \mathbb{R}^{N} \rightarrow(0,+\infty]$ by

$$
d_{\omega}(x):=\min \{|s|: x+s \omega \notin \Omega\} .
$$

Define the pseudodistances $a_{m}: \mathbb{R}^{N} \rightarrow(0,+\infty]$ for $1 \leq m \in \mathbb{R}$ by

$$
a_{m}(x)=\left[\int_{S^{N-1}} d_{\omega}(x)^{-2 m} \mathrm{~d}^{N-1} \omega\right]^{-1 / 2 m},
$$

where $\mathrm{d}^{N-1} \omega$ is the normalized surface measure on the unit spherical shell $S^{N-1}$.

Davies [2] proves the operator inequality

$$
\left.(-\Delta)\right|_{\mathrm{DIR}} \geq \frac{N}{4 a_{1}^{2}}
$$

in the quadratic form sense, thus comparing the Dirichlet Laplacian with a multiplication operator which is large near the boundary of the region. In Theorem 5 we generalize this to a Hardy-Rellich inequality

$$
\left.(-\Delta)^{m}\right|_{\mathrm{DIR}} \geq \frac{(N+2 m-2)(N+2 m-4) \ldots N(2 m-1)(2 m-3) \ldots 1}{4^{m} a_{m}^{2 m}}
$$


for the polyharmonic operators $\left.(-\Delta)^{m}\right|_{\mathrm{DIR}}=H_{\Omega, m}$ acting in $L^{2}(\Omega)$. As a special case, if $\Omega$ is convex we see that

$$
\left.(-\Delta)^{m}\right|_{\mathrm{DIR}} \geq \frac{(2 m-1)^{2}(2 m-3)^{2} \cdots 1^{2}}{4^{m} d^{2 m}}
$$

where

$$
d(x):=\min \{|y-x|: y \notin \Omega\} .
$$

More generally, for regular regions, where the pseudodistances $a_{m}$ are comparable with the distance $d$, a similar inequality is valid. The constants in inequalities (7) and (8) are shown to be optimal.

In both [3] and [4, section 1.9], Davies uses the Hardy inequality (6) to find an upper bound on the trace of the semigroup $e^{-H_{\Omega, 1} t}$. Using the technique of decomposing a region with finite inradius into dyadic cubes he finds a similar lower bound. More explicitly,

$$
(8 \pi t)^{-N / 2} \int_{\Omega} \exp \left[-8 \pi^{2} N^{2} t / d^{2}\right] \leq \operatorname{tr}\left[e^{-H_{\Omega, 1} t}\right] \leq(2 \pi t)^{-N / 2} \int_{\Omega} \exp \left[-N t / 8 a_{1}^{2}\right]
$$

For regular regions $\Omega$ this yields an immediate equivalent condition for $\operatorname{tr}\left[e^{-H_{\Omega, 1} t}\right]$ to be finite, and, as a corollary, a condition for $\operatorname{tr}\left[H_{\Omega, 1}^{-\gamma}\right]$ to be finite.

In Sections 4 and 5 we generalize inequality (9) with some restrictions (see Condition 19 and dependent results), to

$b_{m, N} t^{-N / 2 m} \int_{\Omega} \exp \left[-c_{m, N} t d^{-2 m}\right] \leq \operatorname{tr}\left[e^{-H_{\Omega, m} t}\right] \leq b_{m, N}^{\prime} t^{-N / 2 m} \int_{\Omega} \exp \left[-c_{N, m}^{\prime} t a_{m}^{-2 m}\right]$,

where $b_{m, N}, c_{m, N}, b_{m, N}^{\prime}$ and $c_{m, N}^{\prime}$ are positive constants. This yields an equivalent condition for finite trace of both $e^{-H_{\Omega, m} t}$ and $H_{\Omega, m}^{-\gamma}$.

\section{ACKNOWLEDGMENTS}

I wish to thank E B Davies for suggesting this problem and for his invaluable guidance and support during my research. I thank also Mark Ashbaugh for a useful comment. This research was funded by an EPSRC studentship.

\section{The Hardy-Rellich Operator Inequality}

Our starting point is a one dimensional version of the Hardy-Rellich inequality in the $L^{2}$ setting. For $m=1$ and $m=2$, the following lemma respectively resembles inequalities (11) and (2), where we set $p=2$. 
Lemma 3: Let $\Omega$ be an open (not necessarily connected) set in $\mathbb{R}$. Then

$$
\frac{(2 m-1)^{2}(2 m-3)^{2} \ldots 1^{2}}{4^{m}} \int_{\Omega} \frac{|f(x)|^{2}}{d(x)^{2 m}} \mathrm{~d} x \leq \int_{\Omega}\left|f^{(m)}(x)\right|^{2} \mathrm{~d} x
$$

for all $f \in C_{c}^{\infty}(\Omega)$.

Proof: We prove the statement only for open intervals $(a, b) \subseteq \mathbb{R}$. Suppose that the above statement is true for some $m$. Then applying [5, Lemma 5.3.1] with $\alpha=-2 m$,

$$
\begin{aligned}
\frac{(2 m+1)^{2}}{4} & \int_{a}^{b} \frac{|f(x)|^{2}}{d(x)^{2(m+1)}} \mathrm{d} x \\
& =\frac{(1+2 m)^{2}}{4} \int_{0}^{(b-a) / 2} x^{-2 m-2}|f(x+a)|^{2} \mathrm{~d} x \\
& \quad+\frac{(1+2 m)^{2}}{4} \int_{0}^{(b-a) / 2} x^{-2 m-2}|f(b-x)|^{2} \mathrm{~d} x \\
& \leq \int_{0}^{(b-a) / 2} x^{-2 m}\left|f^{\prime}(x+a)\right|^{2} \mathrm{~d} x+\int_{0}^{(b-a) / 2} x^{-2 m}\left|f^{\prime}(b-a)\right|^{2} \mathrm{~d} x \\
& =\int_{a}^{b} \frac{\left|f^{\prime}(x)\right|^{2}}{d(x)^{2 m}} \mathrm{~d} x \\
& \leq \frac{4^{m}}{(2 m-1)^{2}(2 m-3)^{2} \ldots 1^{2}} \int_{a}^{b}\left|f^{(m+1)}(x)\right|^{2} \mathrm{~d} x .
\end{aligned}
$$

The first step of induction is dealt with by [5, Corollary 5.3.2].

Lemma 4: Let $\omega \in S^{N-1}$. Then

$$
\int_{S^{N-1}}\langle\xi, \omega\rangle^{2 m} \mathrm{~d}^{N-1} \omega=\frac{(2 m-1)(2 m-3) \ldots 1}{(N+2 m-2)(N+2 m-4) \ldots N}|\xi|^{2 m} .
$$

Proof: Since the above integral is rotationally invariant and homogeneous of degree $2 m$ with respect to $\xi$ we see that

$$
\int_{S^{N-1}}\langle\xi, \omega\rangle^{2 m} \mathrm{~d}^{N-1} \omega=c|\xi|^{2 m} .
$$

Setting $\xi=(1,0, \ldots, 0)$ we see that for $N \geq 3$

$$
\begin{aligned}
c & =\int_{S^{N-1}}\langle\xi, \omega\rangle^{2 m} \mathrm{~d}^{N-1} \omega \\
& =\frac{1}{\omega_{N-1}} \int_{-\pi}^{\pi} \int_{0}^{\pi} \cdots \int_{0}^{\pi} \cos ^{2 m} \theta_{1} \sin ^{N-2} \theta_{1} \ldots \sin \theta_{N-2} \mathrm{~d} \theta_{1} \ldots \mathrm{d} \theta_{N-1}
\end{aligned}
$$




$$
=\frac{(2 m-1)(2 m-3) \ldots 1}{(N+2 m-2)(N+2 m-4) \ldots N}
$$

where $\omega_{N-1}$ denotes the surface area of the unit spherical shell $S^{N-1}$ regarded as a subset of $\mathbb{R}^{N}$. The last step of this calculation requires elementary analysis and is therefore omitted. The cases $N=1,2$ are simple.

We may now prove the Hardy-Rellich operator inequality:

Theorem 5: Let $\left.(-\Delta)^{m}\right|_{\text {DIR }}=H_{\Omega, m}$ be the polyharmonic operator of order $2 m$ acting in $L^{2}(\Omega)$, where $\Omega$ is a region in $\mathbb{R}^{N}$, and let $a_{m}$ be the corresponding pseudodistance. Then, in the quadratic form sense,

$$
\left.(-\Delta)^{m}\right|_{\mathrm{DIR}} \geq \frac{(N+2 m-2)(N+2 m-4) \ldots N(2 m-1)(2 m-3) \ldots 1}{4^{m} a_{m}^{2 m}} .
$$

Proof: Let $f \in C_{c}^{\infty}(\Omega)$. Let $\omega \in S^{N-1}$ be fixed, and let $\left\{u_{1}=\omega, u_{2}, \ldots, u_{N}\right\}$ be an orthonormal basis of $\mathbb{R}^{N}$. Let $v=\left(v_{1}, \ldots, v_{N}\right)$ denote coordinates with respect to that basis and let $P$ be the coordinate transition matrix $x=v P$ from $v$ coordinates to standard coordinates. Let $\hat{v}=\left(v_{2}, \ldots, v_{N}\right)$ be fixed, and let $\Omega_{\hat{v}}$ be the open (not necessarily connected) set

$$
\Omega_{\hat{v}}=\left\{v_{1} \in \mathbb{R}: v P \in \Omega\right\} .
$$

Define $g_{\hat{v}}$ and $d_{\hat{v}}$ by

$$
\begin{aligned}
g_{\hat{v}}\left(v_{1}\right) & :=f(v P) \\
d_{\hat{v}}\left(v_{1}\right) & :=d_{\omega}(v P) .
\end{aligned}
$$

Then $g_{\hat{v}} \in C_{c}^{\infty}\left(\Omega_{\hat{v}}\right)$ and

$$
d_{\hat{v}}\left(v_{1}\right)=\min \left\{\left|y-v_{1}\right|: y \notin \Omega_{\hat{v}}\right\}
$$

Using Lemma 3,

$$
\frac{(2 m-1)^{2}(2 m-3)^{2} \ldots 1^{2}}{4^{m}} \int_{\Omega_{\hat{v}}} \frac{\left|g_{\hat{v}}\left(v_{1}\right)\right|^{2}}{d_{\hat{v}}\left(v_{1}\right)^{2 m}} \mathrm{~d} v_{1} \leq \int_{\Omega_{\hat{v}}}\left|g_{\hat{v}}^{(m)}\left(v_{1}\right)\right|^{2} \mathrm{~d} v_{1},
$$

and hence

$$
\begin{aligned}
& \frac{(2 m-1)^{2}(2 m-3)^{2} \ldots 1^{2}}{4^{m}} \int_{\Omega} \frac{|f(x)|^{2}}{a_{m}(x)^{2 m}} \mathrm{~d}^{N} x \\
& =\frac{(2 m-1)^{2}(2 m-3)^{2} \ldots 1^{2}}{4^{m}} \int_{S^{N-1}} \int_{\Omega} \frac{|f(x)|^{2}}{d_{\omega}(x)^{2 m}} \mathrm{~d}^{N} x \mathrm{~d}^{N-1} \omega
\end{aligned}
$$




$$
\begin{aligned}
& =\frac{(2 m-1)^{2}(2 m-3)^{2} \ldots 1^{2}}{4^{m}} \int_{S^{N-1}} \int_{\mathbb{R}^{N-1}} \int_{\Omega_{\hat{v}}} \frac{\left|g_{\hat{v}}\left(v_{1}\right)\right|^{2}}{d_{\hat{v}}\left(v_{1}\right)^{2 m}} \mathrm{~d} v_{1} \mathrm{~d}^{N-1} \hat{v} \mathrm{~d}^{N-1} \omega \\
& \leq \int_{S^{N-1}} \int_{\mathbb{R}^{N-1}} \int_{\Omega_{\hat{v}}}\left|g_{\hat{v}}^{(m)}\left(v_{1}\right)\right|^{2} \mathrm{~d} v_{1} \mathrm{~d}^{N-1} \hat{v} \mathrm{~d}^{N-1} \omega \\
& =\int_{S^{N-1}} \int_{\Omega}\left|\partial_{\omega}^{m} f(x)\right|^{2} \mathrm{~d}^{N} x \mathrm{~d}^{N-1} \omega \\
& =\int_{S^{N-1}} \int_{\mathbb{R}^{N}}\langle\xi, \omega\rangle^{2 m}|\hat{f}(\xi)|^{2} \mathrm{~d}^{N} \xi \mathrm{d}^{N-1} \omega \\
& =\frac{(2 m-1)(2 m-3) \ldots 1}{(N+2 m-2)(N+2 m-4) \ldots N} \int_{\mathbb{R}^{N}}|\xi|^{2 m}|\hat{f}(\xi)|^{2} \mathrm{~d}^{N} \xi \\
& =\frac{(2 m-1)(2 m-3) \ldots 1}{(N+2 m-2)(N+2 m-4) \ldots N} Q_{m}(f) .
\end{aligned}
$$

Corollary 6: Suppose that $\Omega$ is a convex region in $\mathbb{R}^{N}$. Then

$$
\left.(-\Delta)^{m}\right|_{\mathrm{DIR}} \geq \frac{(2 m-1)^{2}(2 m-3)^{2} \cdots 1^{2}}{4^{m} d^{2 m}}
$$

Proof: Let $x \in \Omega$ and let $y \in \partial \Omega$ be such that $|y-x|=d(x)$. Suppose $z \in \Omega$. Constructing the point

$$
p=y+\frac{\langle z-y, x-y\rangle}{\langle z-y, z-y\rangle}(z-y)
$$

we see that

$$
|p-x|^{2}=|y-x|^{2}-\frac{\langle z-y, x-y\rangle^{2}}{\langle z-y, z-y\rangle}
$$

so either

$$
\langle z-y, x-y\rangle=0
$$

or

$$
|p-x|<d(x)
$$

In the second case, $p$ will lie in $\Omega$, and so by convexivity the line segment joining $z$ and $p$ lies in $\Omega$. See Figure 1. Since $y \notin \Omega$, it cannot lie on this segment so

$$
\langle z-y, p-y\rangle>0 .
$$

From the definition of $p$, this implies that

$$
\langle z-y, x-y\rangle>0 \text {. }
$$




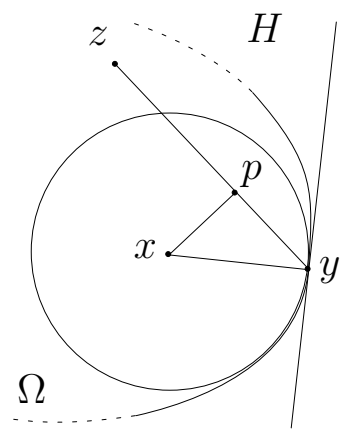

Figure 1: Construction of $p$

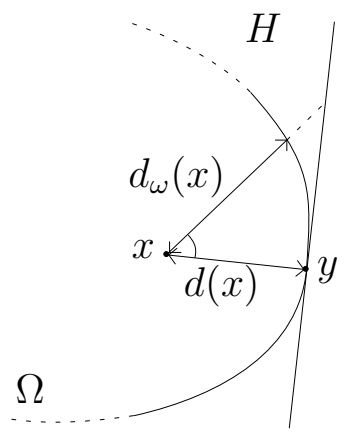

Figure 2: Relationships between distances

In both cases, $z$ lies in the set

$$
\left\{z \in \mathbb{R}^{N}:\langle z-y, x-y\rangle \geq 0\right\} .
$$

Since $\Omega$ is open, it must therefore be a subset of the open half

$$
H:=\left\{z \in \mathbb{R}^{N}:\langle z-y, x-y\rangle>0\right\}
$$

of $\mathbb{R}^{N}$. From the definition (4) of $d_{\omega}$ we see that

$$
\begin{aligned}
d_{\omega}(x)|\langle y-x, \omega\rangle| & \leq \min \{|s|: x+s \omega \notin H\}\left|\left\langle\frac{y-x}{|y-x|}, \omega\right\rangle\right| d(x) \\
& =d(x)^{2} .
\end{aligned}
$$

See Figure 2 for a diagrammatic representation of this last step. Hence

$$
|\langle y-x, \omega\rangle|^{2 m} d(x)^{-4 m} \leq d_{\omega}(x)^{-2 m} .
$$

Therefore

$$
\begin{aligned}
& \frac{(2 m-1)(2 m-3) \ldots 1}{(N+2 m-2)(N+2 m-4) \ldots N} d(x)^{-2 m} \\
& =\frac{(2 m-1)(2 m-3) \ldots 1}{(N+2 m-2)(N+2 m-4) \ldots N}|y-x|^{2 m} d(x)^{-4 m} \\
& =\int_{S^{N-1}}|\langle y-x, \omega\rangle|^{2 m} d(x)^{-4 m} \mathrm{~d}^{N-1} \omega \\
& \leq \int_{S^{N-1}} d_{\omega}(x)^{-2 m} \mathrm{~d}^{N-1} \omega \\
& =a_{m}(x)^{-2 m} \text {. }
\end{aligned}
$$

Using Theorem 5 we see that for $f \in C_{c}^{\infty}(\Omega)$,

$$
\frac{(2 m-1)^{2}(2 m-3)^{2} \ldots 1^{2}}{4^{m}} \int_{\Omega} \frac{|f(x)|^{2}}{d(x)^{2 m}} \mathrm{~d}^{N} x
$$




$$
\begin{aligned}
& \leq \frac{(N+2 m-2)(N+2 m-4) \ldots N(2 m-1)(2 m-3) \ldots 1}{4^{m}} \int_{\Omega} \frac{|f(x)|^{2}}{a_{m}(x)^{2 m}} \mathrm{~d}^{N} x \\
& \leq Q_{m}(f) .
\end{aligned}
$$

Note 7: It is simple to deduce a crude lower bound

$$
\lambda_{1} \geq \frac{(2 m-1)^{2}(2 m-3)^{2} \ldots 1^{2}}{4^{m} \operatorname{Inradius}(\Omega)^{2 m}}
$$

on the first eigenvalue of $\left.(-\Delta)^{m}\right|_{\mathrm{DIR}}$ for regions with finite inradius

$$
\operatorname{Inradius}(\Omega):=\sup _{x \in \Omega} d(x) .
$$

Since the strength of the inequality (14) lies in the values of the potential near the boundary, the constant in the above bound is not sharp.

Note 8: The constants in Theorem 5 and Corollary 6 are optimal. This can be seen by choosing $\Omega=\left\{\left(x_{1}, \ldots, x_{N}\right): x_{1}>0\right\}$ and by considering the sequence of functions $f_{n} \in C_{c}^{\infty}(\Omega)$ defined by

$$
f_{n}(x)=x_{1}^{m-1 / 2} \phi_{n}\left(x_{1}\right) \psi(\hat{x})
$$

where $\psi \in C_{c}^{\infty}\left(\mathbb{R}^{N-1}\right)$ and $\phi_{n} \in C_{c}^{\infty}((0, \infty))$ is chosen so that $\phi_{n}=1$ on the interval $[2 / n, 1], \phi_{n}=0$ on $\mathbb{R} \backslash[1 / n, 2],\left|D^{j} \phi_{n}\right| \leq c n^{j}$ on $[1 / n, 2 / n]$ and $\left|D^{j} \phi_{n}\right| \leq c$ on $[1,2]$, for $j=0,1, \ldots, 2 m$.

Calculations now show that

$$
\int_{\Omega} \frac{\left|f_{n}(x)\right|^{2}}{d(x)^{2 m}} \mathrm{~d}^{N} x \geq \int_{[2 / n, 1] \times \mathbb{R}^{N-1}} x_{1}^{-1}|\psi(\hat{x})|^{2} \mathrm{~d}^{N} x=\|\psi\|_{2}^{2} \ln n / 2
$$

and

$$
Q_{m}\left(f_{n}\right) \leq \frac{(2 m-1)^{2}(2 m-3)^{2} \ldots 1}{4^{m}}\|\psi\|_{2}^{2} \ln n / 2+c^{\prime} .
$$

The constant in Corollary 6 is therefore optimal, and so the constant in Theorem 5 must also be optimal.

\section{Spectral Implications of the Inequality}

In the course of proving Corollary 6 we show, in inequality (17), that the pseudodistance $a_{m}$ is uniformly comparable to the boundary distance function $d$. This motivates the introduction of the following terminology: 
Definition 9: A region $\Omega$ is said to be regular if there is a constant $k<\infty$ such that

$$
d(x) \leq a_{1}(x) \leq k d(x)
$$

for all $x \in \Omega$. More generally, we shall say that a region $\Omega$ is $m$-regular if there is a constant $k_{m}<\infty$ such that

$$
d(x) \leq a_{m}(x) \leq k_{m} d(x)
$$

for all $x \in \Omega$.

Definition 10: We shall say that $\Omega$ satisfies a uniform external ball condition if there exist positive constants $\alpha, \beta$ such that for any $y \in \Omega$ and $0<s \leq \beta$ there exists a ball $B(a ; r)$ with center a satisfying $|a-y| \leq s$, and radius $r$ satisfying $r \geq \alpha$ s, which does not meet $\Omega$.

Examples 11: If any one of the following geometrical conditions is satisfied then the region $\Omega \subseteq \mathbb{R}^{N}$ is regular:

(i) $\Omega$ satisfies a uniform external ball condition with $\beta=\infty$.

(ii) $\Omega$ has finite inradius and satisfies a uniform external ball condition.

(iii) There exists a positive constant c such that

$$
|\{y \notin \Omega:|y-a|<r\}| \geq c r^{N}
$$

for all $a \in \partial \Omega$ and all $r>0$.

Proof: See [4, Theorems 1.5.4 and 1.5.5] and [5, Theorem 5.3.6]. The common characteristic of these situations is that at any point $x \in \Omega$, the directional distance $d_{\omega}(x)$ to the boundary is uniformly comparable to the actual distance $d(x)$ to the boundary over a uniform solid angle.

Lemma 12: Let $x \in \Omega$ be fixed. Then $a_{m}(x)$ is a decreasing function of $m$. Hence if $\Omega$ is regular then it is $m$-regular for all $m \geq 1$.

Proof: Let $\|\cdot\|_{p}$ be norms on the spaces $L^{p}\left(S^{N-1}, \mathrm{~d}^{N-1} \omega\right)$. Since the surface measure $\mathrm{d}^{N-1} \omega$ in Definition 2 is normalized, Hölder's inequality implies that for $m \geq n$

$$
\left\|d_{\omega}(x)^{-1}\right\|_{2 n} \leq\left\|d_{\omega}(x)^{-1}\right\|_{2 m}\|1\|_{2 m n /(m-n)}=\left\|d_{\omega}(x)^{-1}\right\|_{2 m} .
$$

Hence from Definition 2,

$$
a_{m}(x)=\left\|d_{\omega}(x)^{-1}\right\|_{2 m}^{-1} \leq\left\|d_{\omega}(x)^{-1}\right\|_{2 n}^{-1}=a_{n}(x) .
$$


Theorem 13: Suppose that $\Omega$ is m-regular. Then $0 \notin \operatorname{Spec}\left(H_{\Omega, m}\right)$ if and only if the inradius of $\Omega$ is finite.

Proof: Suppose that $\Omega$ has finite inradius. The Hardy-Rellich operator inequality (13) and $m$-regularity (20) imply that

$$
H_{\Omega, m} \geq \frac{(N+2 m-2)(N+2 m-4) \ldots N(2 m-1)(2 m-3) \ldots 1}{4^{m} k_{m}^{2 m} \operatorname{Inradius}(\Omega)^{2 m}} .
$$

Conversely, suppose that $d(x)$ is unbounded. For any $r>0$ there exists a ball $B_{r}$ with radius $r$, contained in $\Omega$. Using the Rayleigh-Ritz variational formula (see [5, Section 4.5]),

$$
0 \leq \min \left(\operatorname{Spec}\left(H_{\Omega, m}\right)\right) \leq \min \left(\operatorname{Spec}\left(H_{B_{r}, m}\right)\right)=r^{-2 m} \min \left(\operatorname{Spec}\left(H_{B_{1}, m}\right)\right) .
$$

Hence $0 \in \operatorname{Spec}\left(H_{\Omega, m}\right)$.

Note that to prove the above theorem one only needs the Hardy inequality for $m=1$ and inequality (3). This approach, however, is not valid for a proof of the following theorem.

Theorem 14: Suppose that $\Omega$ is m-regular. Then $H_{\Omega, m}^{-1}$ is compact if and only if $d(x) \rightarrow 0$ as $x \rightarrow \infty$.

Proof: Using the Hardy-Rellich inequality (13),

$$
\begin{aligned}
H_{\Omega, m} & \geq \frac{1}{2} H_{\Omega, m}+\frac{(N+2 m-2)(N+2 m-4) \ldots N(2 m-1)(2 m-3) \ldots 1}{2.4^{m} a_{m}^{2 m}} \\
& \geq \frac{1}{2} H_{\mathbb{R}^{N}, m}+\frac{(N+2 m-2)(N+2 m-4) \ldots N(2 m-1)(2 m-3) \ldots 1}{2^{2 m+1} k_{m}^{2 m} d^{2 m}}
\end{aligned}
$$

as quadratic forms in $L^{2}\left(\mathbb{R}^{N}\right)$. The last operator in the above inequality has compact resolvent because it is a Schrödinger operator whose potential

$$
V=\frac{(N+2 m-2)(N+2 m-4) \ldots N(2 m-1)(2 m-3) \ldots 1}{2^{2 m+1} k_{m}^{2 m} d^{2 m}}
$$

satisfies $V(x) \rightarrow \infty$ as $|x| \rightarrow \infty$. See [9, Theorem 12.5.5], although this result is proved with the unnecessary restriction that $N<2 m$. Simple modification of the proof of [11, Theorem XIII.67] yields the result without any such restriction. It now follows that $H_{\Omega, m}^{-1}$ is compact.

Conversely suppose $d(x)$ does not converge to zero as $x \rightarrow \infty, x \in \Omega$. Then there exist $r>0$ and a sequence of balls $B_{i} \subseteq \Omega$, each with radius $r$. Let $\phi_{i}$ be the groundstate of the operator $H_{B_{i}, m}$. Then

$$
\left\langle\phi_{i}, \phi_{j}\right\rangle=\delta_{i j}
$$




$$
\left\langle H_{\Omega, m} \phi_{i}, \phi_{j}\right\rangle=c \delta_{i j}
$$

where $c$ is independent of $i, j$. Using the Rayleigh-Ritz formula of section [5] we see that $H_{\Omega, m}^{-1}$ cannot be compact.

\section{Lower Bound on the Trace of the Polyharmonic Semigroup}

In the remaining sections we build upon the methods of Davies [3] to obtain lower and upper bounds on the trace of the semigroup $e^{-H_{\Omega, m} t}$ and the resolvent $H_{\Omega, m}^{-\gamma}$. The proof of the lower bound in Theorem 18 requires the following sequence of lemmas:

Lemma 15: Let $\lambda_{m, n}$ denote the $n$-th eigenvalue of the polyharmonic operator $\left.(-\Delta)^{m}\right|_{\mathrm{DIR}}$ acting in $L^{2}((0,1))$. Then

$$
[n \pi]^{2 m} \leq \lambda_{m, n} \leq[(m+n-1) \pi]^{2 m} .
$$

Proof: The left hand inequality is a consequence of inequality (35). We prove the other inequality as follows: Let $f_{r} \in W_{0}^{m, 2}((0,1))$ be defined by

$$
f_{r}(x)=\sin ^{m-1} \pi x \sin r \pi x .
$$

Then $f_{r} \in M_{r+m-1}$ where

$$
M_{s}=\operatorname{lin}\{1, \sin \pi x, \cos \pi x, \ldots, \sin s \pi x, \cos s \pi x\} .
$$

Let $L_{n} \subseteq W_{0}^{m, 2}([0,1])$ be defined by

$$
L_{n}=\operatorname{lin}\left\{f_{r}: 1 \leq r \leq n\right\} .
$$

Then $L_{n} \subseteq M_{n+m-1}$, and by the Rayleigh-Ritz formula [5],

$$
\begin{aligned}
\lambda_{m, n} & \leq \sup \left\{Q_{m}(f): f \in L_{n},\|f\|_{2}=1\right\} \\
& =\sup \left\{\left\|D^{m} f\right\|_{2}^{2}: f \in L_{n},\|f\|_{2}=1\right\} \\
& \leq \sup \left\{\left\|D^{m} f\right\|_{2}^{2}: f \in M_{n+m-1},\|f\|_{2}=1\right\} .
\end{aligned}
$$

Suppose that $f \in M_{s}$ and $\|f\|_{2}=1$. Then

$$
f(x)=\alpha_{0}+\sum_{r=1}^{s}\left(\alpha_{r} \sqrt{2} \cos r \pi x+\beta_{r} \sqrt{2} \sin r \pi x\right),
$$


where

$$
\sum_{r=0}^{s}\left(\alpha_{r}^{2}+\beta_{r}^{2}\right)=1
$$

Now

$$
\left\|D^{m} f\right\|_{2}^{2}=\sum_{r=1}^{s}\left(\alpha_{r}^{2} .(r \pi)^{2 m}+\beta_{r}^{2} \cdot(r \pi)^{2 m}\right) \leq(s \pi)^{2 m}
$$

Hence by inequality (23),

$$
\lambda_{m, n} \leq[(m+n-1) \pi]^{2 m} .
$$

Lemma 16: The operator

$$
H^{\prime}=\sum_{i=1}^{N}\left(-\frac{\partial^{2}}{\partial x_{i}^{2}}\right)^{m}
$$

acting in $L^{2}(C)$ with Dirichlet boundary conditions, where $C=(0, \delta)^{N}$, is uniformly elliptic, homogeneous of order $2 \mathrm{~m}$, and has compact resolvent. The eigenvalues of $H^{\prime}$ are given by

$$
\mu_{n}=\delta^{-2 m} \sum_{i=1}^{N} \lambda_{m, n_{i}}
$$

where $n=\left(n_{1}, \ldots, n_{N}\right)$ is a non-negative multi-index and $\lambda_{m, n_{i}}$ are the eigenvalues of the one-dimensional polyharmonic operator in Lemma 15.

Proof: Using the Fourier transform we may write the quadratic form $Q^{\prime}$ of the operator $H^{\prime}$ as

$$
Q^{\prime}(f)=\int_{\mathbb{R}^{N}} \sum_{i=1}^{N} \xi_{i}^{2 m}|\hat{f}(\xi)|^{2} \mathrm{~d}^{N} \xi
$$

The symbol of $H^{\prime}$ is

$$
a(x, \xi)=\sum_{i=1}^{N} \xi_{i}^{2 m}
$$

and is homogeneous of degree $2 m$. Since

$$
N^{-(m-1)}|\xi|^{2 m} \leq a(x, \xi) \leq|\xi|^{2 m},
$$


we see that $H^{\prime}$ is uniformly elliptic.

Let $\left\{f_{m, n}\right\}_{n=1}^{\infty}$ be the orthonormal sequence of eigenfunctions corresponding to the eigenvalues $\lambda_{m, n}$ of $\left.(-\Delta)^{m}\right|_{\text {DIR }}$ acting in $L^{2}((0,1))$. For each nonnegative multi-index $n$ define

$$
f_{n}(x)=\delta^{-N / 2} \prod_{i=1}^{N} f_{m, n_{i}}\left(x_{i} / \delta\right) .
$$

Since the functions $f_{m, n}$ form a complete orthonormal set in $L^{2}((0,1))$, the functions $f_{n}$ form a complete orthonormal set in $L^{2}(C)$. Moreover, since the $f_{n}$ are eigenfunctions of $H^{\prime}$ with corresponding eigenvalues $\mu_{n}$, we have found a complete list of eigenvalues.

To find the lower bound on $\operatorname{tr}\left[e^{-H_{\Omega, m} t}\right]$ we use the technique of decomposing $\Omega$ into dyadic cubes by introducing Dirichlet boundary conditions along various internal partitioning surfaces. The cubes $C \subseteq \Omega$ we use are of the form

$$
C=\left\{x \in \mathbb{R}^{N}: \frac{a_{i}}{2^{n}}<x_{i}<\frac{a_{i}+1}{2^{n}}\right\}
$$

for some $n \in \mathbb{Z}$ and some $a \in \mathbb{Z}^{N}$. Ordering the dyadic cubes (30) by inclusion, let $\left\{C_{r}: r \in \mathbb{N}\right\}$ be an enumeration of the maximal cubes contained in $\Omega$, provided at least one exists. Let $\delta_{r}$ be the side length of $C_{r}$ and let $\Omega^{\prime}=\bigcup_{r=1}^{\infty} C_{r}$.

Lemma 17: The cubes $C_{r}$ are disjoint. Suppose that the inradius of $\Omega$ is finite. Then $\overline{\Omega^{\prime}}=\bar{\Omega}$, and moreover for $x \in \overline{C_{r}}$ we have $d(x) \leq 2 N^{1 / 2} \delta_{r}$.

Proof: The inclusion $\overline{\Omega^{\prime}} \subseteq \bar{\Omega}$ is obvious. Conversely, suppose that $x \in \Omega$. Then $\overline{B(x ; d(x))} \subseteq \bar{\Omega}$ and so $x$ will lie in some closed dyadic cube $\bar{C} \subseteq \bar{\Omega}$ with diameter at least $d(x) / 2$. The edge length of such a cube will be at least $d(x) /\left(2 N^{1 / 2}\right)$. Since $d(x)$ is bounded, the point $x$ will lie in a maximal cube $\overline{C_{r}}$ with edge length $\delta_{r} \geq d(x) /\left(2 N^{1 / 2}\right)$. Hence $\Omega \subseteq \overline{\Omega^{\prime}}$ and $d(x) \leq 2 N^{1 / 2} \delta_{r}$.

We shall always assume that $\Omega$ has finite inradius, for otherwise using Theorem 14, we may deduce that $\operatorname{tr}\left[e^{-H_{\Omega, m} t}\right]=\infty$.

Theorem 18: For $0<t<\infty$

$$
b_{m, N} t^{-N / 2 m} \int_{\Omega} \exp \left[-c_{m, N} d^{-2 m} t\right] \leq \operatorname{tr}\left[e^{-H_{\Omega, m} t}\right],
$$

where

$$
b_{m, N}=N^{-N(m-1) / 2 m}(2 \pi)^{-N} \Gamma(1+1 / 2 m) 2^{N / 2 m}
$$


and

$$
c_{m, N}=(4 m N \pi)^{2 m} / 2 .
$$

Proof: Let $\Omega=(0,1) \subseteq \mathbb{R}$. Using the notation of Lemma 15 and the spectral mapping theorem, we see that the trace of the semigroup $e^{-H_{[0,1], m} t}$ is $\sum_{n=1}^{\infty} e^{-\lambda_{m, n} t}$, and moreover that

$$
\begin{aligned}
\sum_{n=1}^{\infty} e^{-\lambda_{m, n} t} & \geq \sum_{n=0}^{\infty} e^{-[(m+n) \pi]^{2 m} t} \\
& \geq \sum_{n=0}^{\infty} e^{-2^{2 m-1}\left(m^{2 m}+n^{2 m}\right) \pi^{2 m} t} \\
& \geq e^{-(2 m \pi)^{2 m} t / 2} \int_{0}^{\infty} e^{(2 x \pi)^{2 m} t / 2} \mathrm{~d} x \\
& =\left[(2 \pi)^{2 m} t / 2\right]^{-1 / 2 m} \Gamma(1+1 / 2 m) e^{-(2 m \pi)^{2 m} t / 2} \\
& =b_{m, 1} t^{-1 / 2 m} \exp \left[-c_{m, 1} 2^{-2 m} t\right] .
\end{aligned}
$$

Let $H^{\prime}$ denote the operator (24) acting in $L^{2}\left(C_{r}\right)$ with Dirichlet boundary conditions. Inequality (28) implies that

$$
H_{C_{r}, m} \leq N^{m-1} H^{\prime},
$$

and hence using Lemma 16 and equation (32),

$$
\begin{aligned}
\operatorname{tr}\left[e^{-H_{C r, m} t}\right] & \geq \operatorname{tr}\left[e^{-N^{m-1} H^{\prime} t}\right] \\
& =\sum_{n \in \mathbb{N}^{N}} e^{-N^{m-1} \delta_{r}^{-2 m} t \sum_{i=1}^{N} \lambda_{m, n_{i}}} \\
& =\sum_{n \in \mathbb{N}^{N}} \prod_{i=1}^{N} e^{-N^{m-1} \delta_{r}^{-2 m} t \lambda_{m, n_{i}}} \\
& =\left[\sum_{n=1}^{\infty} e^{-N^{m-1} \delta_{r}^{-2 m} t \lambda_{m, n}}\right]^{N} \\
& \geq b_{m, 1}^{N}\left[N^{m-1} \delta_{r}^{-2 m} t\right]^{-N / 2 m} \exp \left[-c_{m, 1} 2^{-2 m} N^{m} \delta_{r}^{-2 m} t\right] \\
& =b_{m, N} \delta_{r}^{N} t^{-N / 2 m} \exp \left[-c_{m, N}\left(2 N^{1 / 2} \delta_{r}\right)^{-2 m} t\right] .
\end{aligned}
$$

Using the results of Lemma 17,

$$
\begin{aligned}
\operatorname{tr}\left[e^{-H_{\Omega, m} t}\right] & \geq \operatorname{tr}\left[e^{-H_{\Omega^{\prime}, m^{t}}}\right] \\
& =\sum_{r=1}^{\infty} \operatorname{tr}\left[e^{-H_{C_{r}, m} t}\right]
\end{aligned}
$$




$$
\begin{aligned}
& \geq \sum_{r=1}^{\infty} b_{m, N} \delta_{r}^{N} t^{-N / 2 m} \exp \left[-c_{m, N}\left(2 N^{1 / 2} \delta_{r}\right)^{-2 m} t\right] \\
& =b_{m, N} t^{-N / 2 m} \sum_{r=1}^{\infty} \int_{C_{n}} \exp \left[-c_{m, N} t d(x)^{-2 m}\right] \mathrm{d}^{N} x \\
& =b_{m, N} t^{-N / 2 m} \int_{\Omega} \exp \left[-c_{m, N} t d^{-2 m}\right] .
\end{aligned}
$$

\section{Upper Bound on the Trace of the Polyharmonic Semigroup}

In order to prove an upper bound on the trace we shall need to assume that the region $\Omega$ satisfies the following condition.

Condition 19: Let $\Omega$ be a region such that the kernel $K_{\Omega}(t, x, y)$ of $e^{-H_{\Omega, m} t}$ exists, is jointly continuous and satisfies

$$
\left|K_{\Omega}(t, x, y)\right| \leq c t^{-N / 2 m} .
$$

for some $c=c_{\Omega}$, and for all $t>0$ and $x, y \in \Omega$. Let $b_{m, N}^{\prime}=2^{N / 2 m} c$.

Two special cases in which this condition is satisfied are given in the following two examples:

Example 20: For all $N$ the Laplacian $\left.(-\Delta)\right|_{\mathrm{DIR}}$ acting in $L^{2}(\Omega)$ has a heat kernel $K(t, x, y)$ which satisfies

$$
0 \leq K(t, x, y) \leq(4 \pi t)^{-N / 2}
$$

for all $x, y \in \Omega$ and $t>0$.

Proof: See [4, example 2.1.8].

Example 21: Suppose that $\Omega \subseteq \mathbb{R}^{N}$ and $N<2 m$. Then $\left.(-\Delta)^{m}\right|_{\text {DIR }}$ acting in $L^{2}(\Omega)$ has a heat kernel which satisfies

$$
|K(t, x, y)| \leq c t^{-N / 2 m}
$$

for all $x, y \in \Omega$ and all $t>0$.

Proof: By the spectral mapping theorem we see that

$$
\left\|H_{\Omega, m}^{1 / 2} e^{-H_{\Omega, m} t}\right\|_{2,2} \leq c t^{-1 / 2} .
$$

For $f \in L^{2}(\Omega)$ and $t>0$, let $f_{t}=e^{-H_{\Omega, m} t} f \in W_{0}^{m, 2}(\Omega)$. Using a standard Sobolev embedding theorem,

$$
\left\|f_{t}\right\|_{\infty} \leq c\left\|H_{\Omega, m}^{1 / 2} f_{t}\right\|^{N / 2 m}\left\|f_{t}\right\|_{2}^{1-N / 2 m}
$$




$$
\begin{aligned}
& \leq c\left\|H_{\Omega, m}^{1 / 2} e^{-H_{\Omega, m} t}\right\|_{2,2}^{N / 2 m}\|f\|_{2}^{N / 2 m}\|f\|_{2}^{1-N / 2 m} \\
& \leq c t^{-N / 4 m}\|f\|_{2} .
\end{aligned}
$$

Hence

$$
\left\|e^{-H_{\Omega, m} t}\right\|_{\infty, 2} \leq c t^{-N / 4 m}
$$

By duality,

$$
\left\|e^{-H_{\Omega, m} t}\right\|_{\infty, 1} \leq\left\|e^{-H_{\Omega, m} t}\right\|_{\infty, 2}\left\|e^{-H_{\Omega, m} t}\right\|_{2,1} \leq c t^{-N / 2 m} .
$$

Define $\phi_{t}: \Omega \rightarrow L^{2}(\Omega) \cap L^{\infty}(\Omega)$ by the property

$$
\left\langle f, \phi_{t}(x)\right\rangle=\left(e^{-H_{\Omega, m} t} f\right)(x)
$$

for all $f \in L^{1}(\Omega) \cap L^{2}(\Omega)$. Since $e^{-H_{\Omega, m} t} f$ is a smooth function, the map $x \mapsto \phi_{t}(x)$ from $\Omega \rightarrow L^{2}(\Omega)$ is smooth in the weak Hilbert space sense and hence, by [1, Corollary 1.42], it is smooth. Define

$$
K(t, x, y)=\left[\phi_{t}(x)\right](y)
$$

Then by the definition of $\phi_{t}$, we see that $K(t, .,$.$) is an integral kernel of$ $e^{-H_{\Omega, m} t}$. Using the identity

$$
\left[\phi_{s+t}(x)\right](y)=\left\langle\phi_{s}(x), \phi_{t}(y)\right\rangle
$$

for all $t, s>0$ we see that $K$ is smooth in $x$ and $y$. Moreover, by (34) we see that

$$
|K(t, x, y)| \leq c t^{-N / 2 m}
$$

Theorem 22: Suppose that $\Omega$ satisfies Condition [19. Then

$$
\operatorname{tr}\left[e^{-H_{\Omega, m} t}\right] \leq b_{m, N}^{\prime} t^{-N / 2 m} \int_{\Omega} \exp \left[-c_{N, m}^{\prime} a_{m}^{-2 m} t\right]
$$

where $b_{m, N}^{\prime}$ is determined by Condition 19, and

$$
c_{m, N}^{\prime}=2^{-2 m-1}(N+2 m-2)(N+2 m-4) \ldots N(2 m-1)(2 m-3) \ldots 1 .
$$

Proof: The Hardy-Rellich inequality (13) shows that

$$
H_{\Omega, m} \geq \frac{1}{2} H_{\Omega, m}+\frac{(N+2 m-2)(N+2 m-4) \ldots N(2 m-1)(2 m-3) \ldots 1}{2.4^{m} a_{m}^{2 m}} .
$$


Using the Golden-Thompson inequality [8], integration of the kernel along the diagonal [12, pages 65,66], and Condition 19 we see that

$$
\begin{aligned}
\operatorname{tr}\left[e^{-H_{\Omega, m} t}\right] & \leq \operatorname{tr}\left[\exp \left[-H_{\Omega, m} t / 2-c_{N, m}^{\prime} a_{m}^{-2 m} t\right]\right] \\
& \leq \operatorname{tr}\left[e^{-H_{\Omega, m} t / 4} \exp \left[-c_{N, m}^{\prime} a_{m}^{-2 m} t\right] e^{-H_{\Omega, m} t / 4}\right] \\
& =\int_{\Omega} K_{\Omega}(t / 2, x, x) \exp \left[-c_{N, m}^{\prime} a_{m}(x)^{-2 m} t\right] \mathrm{d}^{N} x \\
& \leq b_{m, N}^{\prime} t^{-N / 2 m} \int_{\Omega} \exp \left[-c_{N, m}^{\prime} a_{m}(x)^{-2 m} t\right] \mathrm{d}^{N} x .
\end{aligned}
$$

\section{Equivalent Conditions for Finite Trace}

We can now use the lower and upper bounds of Theorems 18 and 22 to give conditions for finite trace of $e^{-H_{\Omega, m} t}$ and $H_{\Omega, m}^{-\gamma}$ in terms of integrals involving the distance function $d$.

Theorem 23: Suppose that $\Omega$ is m-regular and satisfies Condition 19. Then

$$
\operatorname{tr}\left[e^{-H_{\Omega, m} t}\right]<\infty
$$

for all $t \in(0, \infty)$ if and only if

$$
\int_{\Omega} e^{-t d^{-2 m}}<\infty
$$

for all $t \in(0, \infty)$.

Proof: Since $\Omega$ is $m$-regular, inequality (10) becomes

$$
b t^{-N / 2 m} \int_{\Omega} \exp \left[-c t d^{-2 m}\right] \leq \operatorname{tr}\left[e^{-H_{\Omega, m} t}\right] \leq b^{\prime} t^{-N / 2 m} \int_{\Omega} \exp \left[-c^{\prime} t k_{m} d^{-2 m}\right] .
$$

Corollary 24: Suppose that $\Omega$ is m-regular and satisfies Condition 19, and that $\gamma>N / 2 m$. Then

$$
\operatorname{tr}\left[H_{\Omega, m}^{-\gamma}\right]<\infty
$$

if and only if

$$
\int_{\Omega} d^{2 m \gamma-N}<\infty
$$


Proof: Using Fubini's theorem for traces we see that

$$
\begin{aligned}
\int_{0}^{\infty} \operatorname{tr}\left[e^{-H_{\Omega, m} t}\right] t^{\gamma-1} \mathrm{~d} t & =\operatorname{tr}\left[\int_{0}^{\infty} e^{-H_{\Omega, m} t} t^{\gamma-1} \mathrm{~d} t\right] \\
& =\Gamma(\gamma) \operatorname{tr}\left[H_{\Omega, m}^{-\gamma}\right]
\end{aligned}
$$

Integration of inequality (35) gives

$$
\begin{aligned}
\int_{0}^{\infty} \operatorname{tr}\left[e^{-H_{\Omega, m} t}\right] t^{\gamma-1} \mathrm{~d} t & \leq \int_{\Omega} \int_{0}^{\infty} b^{\prime} t^{-N / 2 m+\gamma-1} \exp \left[-c^{\prime} t a_{m}(x)^{-2 m}\right] \mathrm{d} t \mathrm{~d}^{N} x \\
& =b^{\prime} c^{\prime-\gamma+N / 2 m} \Gamma(\gamma-N / 2 m) \int_{\Omega} a_{m}^{2 m \gamma-N}
\end{aligned}
$$

and similarly by integrating inequality (31) we see that

$$
b c^{-\gamma+N / 2 m} \Gamma(\gamma-N / 2 m) \int_{\Omega} d^{2 m \gamma-N} \leq \int_{0}^{\infty} \operatorname{tr}\left[e^{-H_{\Omega, m} t}\right] t^{\gamma-1} \mathrm{~d} t .
$$

The result follows as in Theorem 23 because $\Omega$ is $m$-regular.

\section{REFERENCES}

[1] E. B. Davies. One-parameter semigroups. Academic Press, 1980.

[2] E. B. Davies. Some norm bounds and quadratic form inequalities for Schrödinger operators II. Journal of Operator Theory, 12:177-196, 1984.

[3] E. B. Davies. Trace properties of the Dirichlet Laplacian. Math. Zeit., 188:245-251, 1985.

[4] E. B. Davies. Heat kernels and spectral theory. Cambridge University Press, 1989.

[5] E. B. Davies. Spectral theory and differential operators. Cambridge University Press, 1995.

[6] E. B. Davies and A. M. Hinz. Explicit constants for Rellich inequalities in $L_{p}(\Omega)$. Math. Zeit., to appear.

[7] G. H. Hardy. Note on a theorem of Hilbert. Math. Zeit., 6:314-317, 1920.

[8] A. Lenard. Generalization of the Golden-Thompson inequality. Indiana Univ. J. Math., 21:457-468, 1971. 
[9] V. G. Maz'ya. Sobolev Spaces. Springer-Verlag, 1985.

[10] B. Opic and A. Kufner. Hardy-type inequalities. Longman Scientific \& Technical, 1990.

[11] M. Reed and B. Simon. Methods of modern mathematical physics, volume IV: Analysis of operators. Academic press, 1978.

[12] M. Reed and B. Simon. Methods of modern mathematical physics, volume III: Scattering Theory. Academic press, 1979.

[13] F. Rellich. Halbbeschränkte Differentialoperatoren höherer Ordnung. In J. C. H. Gerretsen and J. de Groot, editors, Proceedings of the International Congress of Mathematicians 1954, volume III, pages 247-249, 1956.

M. Owen: Erwin Schrödinger International Institute for Mathematical Physics, Boltzmanngasse 9, A-1090 Wien, Austria.

E-mail address: mowen@esi.ac.at 